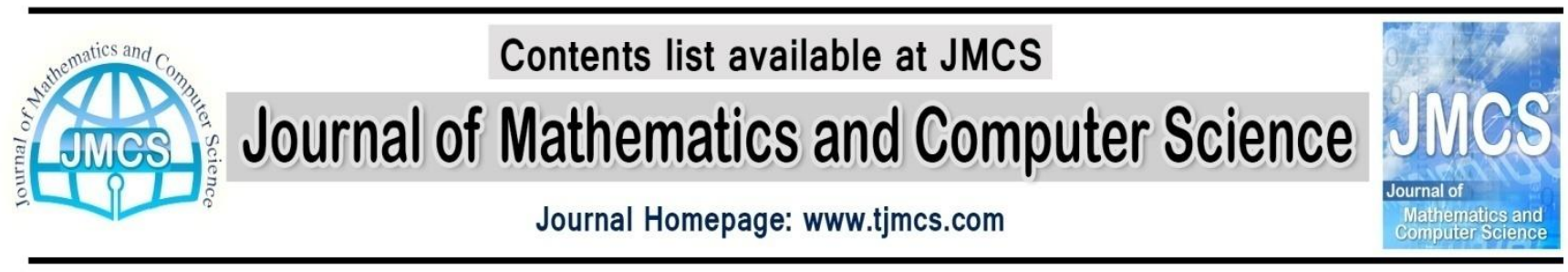

\title{
Connections of Linear Operators defined by Analytic Functions with $\boldsymbol{Q}_{\boldsymbol{p}}$ Spaces
}

\author{
Z. Orouji, R. Aghalary, A. Ebadian \\ Department of Mathematics, Faculty of Science, Urmia University, Urmia, Iran \\ z.oroujy@yahoo.com \\ raghalary@yahoo.com and r.aghalary@urmia.ac.ir \\ a.ebadian@urmia.ac.ir
}

Article history:

Received January 2014

Accepted March 2014

Available online March 2014

\begin{abstract}
This paper is concerned mainly with the linear operators $I_{f}^{\gamma, \alpha}$ and $J_{f}^{\gamma, \alpha}$ of analytic function $f$.The norm of $I_{f}^{\gamma, \alpha}$ and $J_{f}^{\gamma, \alpha}$ on some analytic function spaces is computed in this paper. We study the relation between $I_{f}^{\gamma, \alpha}$ and $J_{f}^{\gamma, \alpha}$ operators, the $B(\lambda)$ spaces and $Q_{p}$ spaces $(0<p<\infty)$.
\end{abstract}

Keywords: Integral operator, $\mathrm{Q}_{\mathrm{p}}$ spaces, Pre-Schwarzian derivative.

\section{Introduction}

Let $\mathcal{H}(\mathbb{D})$ denote the class of analytic functions fon the open unit $\operatorname{disc\mathbb {D}}=\{z \in \mathbb{C}:|z|<1\}$. Also denote by $\mathcal{A}$ the subclass of $\mathcal{H}(\mathbb{D})$ consisting of functions normalized by

$$
f(z)=z+\sum_{n=2}^{\infty} a_{n} z^{n}
$$

and $\mathcal{S}$ the class of all univalent functions in $\mathcal{A}$.

A function $f \in \mathcal{H}(\mathbb{D})$ is a Bloch function if

$$
\|f\|_{B}:=|f(0)|+\sup _{z \in \mathbb{D}}\left(1-|z|^{2}\right)\left|f^{\prime}(z)\right|<\infty
$$

The space of all Bloch functions is denoted by $\mathbb{B}$. A classical source for Bloch functions is $[18,19]$.

For $\alpha>0$, the $\alpha$-Bloch space, denoted by $\mathbb{B}^{\alpha}$, is the space of all functions $f$ in $\mathbb{D}$, for which

$$
\|f\|_{\mathbb{B}^{\alpha}}:=|f(0)|+\sup _{z \in \mathbb{D}}\left|f^{\prime}(z)\right|\left(1-|z|^{2}\right)^{\alpha}<\infty .
$$

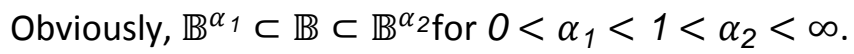

The Hardy space $H^{p}(0<p<\infty)$ is the class of all functions $f$ analytic in $\mathbb{D}$ such that 


$$
\|f\|_{p}:=\lim _{r \rightarrow 1^{-}} M_{p}(r, f)<\infty,
$$

where

$$
M_{p}(r, f)=\left(\frac{1}{2 \pi} \int_{0}^{2 \pi}\left|f\left(r e^{i \theta}\right)\right|^{p} d \theta\right)^{\frac{1}{p}}, \quad(0<p<\infty)
$$

and

$$
M_{\infty}(r, f)=\max _{|z| \leq r}|f(z)| .
$$

we mention [4] as a general reference for the theory of Hardy spaces.

The space $B M O A$ consists of those functions $f$ in $H^{1}$ whose boundary values have bounded mean oscillation in the unit circle $\partial \mathbb{D}$ as defined by $F$. John and L. Nirenberg [10]. We mention [5, 6, 14], as general references for these space. Let us recall that

$$
H^{\infty} \subset B M O A \subset \bigcap_{0<p<\infty} H^{p}, \quad H^{\infty} \subset B M O A \subset \mathbb{B} .
$$

Even though the inclusion $B M O A \subset \mathbb{B}$ is strict, a result of Pommerenke [13] implies that

$$
S \cap \mathbb{B}=\boldsymbol{S} \cap \boldsymbol{B M O A}(1.1)
$$

Let $d \sigma$ denote the normalized Lebesgue area measure in $\mathbb{D}$ and $g(a, z)$ the Green function with logarithmic singularity at $a$, i.e., $g(a, z)=-\log \left|\varphi_{a}(z)\right|$, where $\varphi_{a}(z)=(a-z) /(1-\bar{a} z)$ is the Mobius transformation of $\mathbb{D}$.

For $0<p<\infty, Q_{p}$ is the space of all functions $f \in \mathcal{H}(\mathbb{D})$, for which

$$
\|f\|_{Q_{p}}^{2}=|f(0)|^{2}+\sup _{a \in \mathbb{D}} \int_{D}\left|f^{\prime}(z)\right|^{2}\left(1-\left|\varphi_{a}(z)\right|^{2}\right)^{p} d \sigma(z)<\infty .
$$

This space was introduced by Aulaskari and Lappan in [1] while looking for new characterizations of Bloch functions, and we mention the books [16] and [17] as general references for the space $Q_{p}$. Let us just mention here that $Q_{p}=\mathbb{B}$ for all $p>1 ; Q_{1}=B M O A$; and that whenever $0<p<1, Q_{p}$ is a proper subspace of BMOA.Aulaskari, Lappan, Xiao, and Zhao[2] extended (1.1) showing that

$\boldsymbol{S} \cap \boldsymbol{Q}_{\boldsymbol{p}}=\boldsymbol{S} \cap \mathbb{B}, \quad(0<p<\infty)$.

For any $f \in \mathcal{H}(\mathbb{D})$, the next two integral operators on $\mathcal{H}(\mathbb{D})$ are induced as follows:

$$
I_{f}^{\gamma, \alpha}(h)(z)=\int_{0}^{z} h^{\prime}(w) f^{\gamma}(w) w^{\alpha-1} d w, \quad(z \in \mathbb{D}),
$$

and

$$
J_{f}^{\gamma, \alpha}(h)(z)=\int_{0}^{z} h(w) f^{\prime \gamma}(w) w^{\alpha-1} d w, \quad(z \in \mathbb{D})
$$

where $\gamma, \alpha>0$.

If $\gamma=\alpha=1$, then $I_{f}^{1,1}(h)=I_{f}(h)$ and $J_{f}^{1,1}(h)=J_{f}(h)$, which are the Alexander operators and Both integral operators have been studied by many authors. See $[12,13,16,18,19]$ and the references therein.

Norm of composition operator, weighted composition operator and some integral operators have been studied extensively by many authors, see $[7,15]$. 


\section{Main Results}

In this section, we state and prove our main results. In order to formulate our main results, we need the following lemma from ([11], Lemma 2.1).

Lemma 2.1.Let $0<p<1$. For any $z_{0} \in \mathbb{D}$, the function

$$
g_{z_{0}}(z)=\frac{z_{0}-z}{1-\overline{z_{0}} Z}-z_{0}
$$

is analytic in $\mathbb{D}$ and $\left\|g_{z_{0}}\right\|_{Q_{p}}=1 /(p+1)^{1 / 2}$.

Theorem 2.1.Let $0<p<1$ and $\alpha+\gamma \geq 1$. If $f \in \mathcal{A}$, then $I_{f}^{\gamma, \alpha}$ is bounded on $Q_{p}$ space if and only if $f \in H^{\infty}$. Moreover, $\left\|I_{f}^{\gamma, \alpha}\right\|_{Q_{p}}=\left\|I f^{\gamma}\right\|_{H^{\infty}}$ where $I(z)=z^{\alpha-1}$.

Proof. Let $f \in H^{\infty}$ and $M:=\|f\|_{H^{\infty}}$. Then by Showarz lemma, for any $z \in \mathbb{D}$, we have

$$
\begin{aligned}
& \frac{|f(z)|}{M}<|z| \Longrightarrow \\
& \left|z^{\alpha-1} f^{\gamma}(z)\right|<M|z|^{\alpha+\gamma-1} \leq M .
\end{aligned}
$$

Therefore $z^{\alpha-1} f^{\gamma}(z) \in H^{\infty}$ and there exists $C>0$ such that $\left\|I f^{\gamma}\right\|_{H^{\infty}}=C$.

Now for any $\|h\|_{Q_{p}}=1$, we have

$$
\begin{aligned}
\left\|I_{f}^{\gamma, \alpha} h\right\|^{2} & =\sup _{a \in \mathbb{D}} \int_{D}\left|h^{\prime}(z) z^{\alpha-1} f^{\gamma}(z)\right|^{2}\left(1-\left|\varphi_{a}(z)\right|^{2}\right)^{p} d \sigma(z) \\
& \leq\left\|I f^{\gamma}\right\|_{H^{\infty}}^{2} \sup _{a \in \mathbb{D}} \int_{D}\left|h^{\prime}(z)\right|^{2}\left(1-\left|\varphi_{a}(z)\right|^{2}\right)^{p} d \sigma(z) \\
& \leq C^{2}\|h\|_{Q_{p}} \\
& =C^{2}
\end{aligned}
$$

Then $\left\|I_{f}^{\gamma, \alpha}\right\|_{Q_{p}} \leq C$.To prove the converse, for given any $\epsilon>0$, there existsz $z_{0} \in \mathbb{D}$ such that

$\left|I\left(z_{0}\right) f^{\gamma}\left(z_{0}\right)\right|>C-\epsilon$. Let

$$
h(z)=\frac{g_{z_{0}}(z)}{\left\|g_{z_{0}}\right\|_{Q_{p}}},
$$

where

$$
g_{z_{0}}(z)=\frac{z_{0}-z}{1-\bar{z}_{0} z}-z_{0}
$$

It is easy to see that

Therefore we have

$$
\|h\|_{Q_{p}}=1, \quad\left|h^{\prime}\left(z_{0}\right)\right|\left(1-\left|z_{0}\right|^{2}\right)=1 /\left\|g_{z_{0}}\right\|_{Q_{p}}
$$




$$
\begin{aligned}
\left\|I_{f}^{\gamma, \alpha}\right\|^{2} & \geq\left\|I_{f}^{\gamma, \alpha} h\right\|_{Q_{p}}^{2}=\sup _{a \in \mathbb{D}} \int_{D}\left|h^{\prime}(z) z^{\alpha-1} f^{\gamma}(z)\right|^{2}\left(1-\left|\varphi_{a}(z)\right|^{2}\right)^{p} d \sigma(z) \\
& =\sup _{a \in \mathbb{D}} \int_{D}\left|h^{\prime}\left(\varphi_{a}(w)\right) \varphi_{a}^{\alpha-1}(w) f^{\gamma}\left(\varphi_{a}(w)\right) \varphi^{\prime}{ }_{a}(w)\right|^{2}\left(1-|w|^{2}\right)^{p} d \sigma(w) .
\end{aligned}
$$

Taking $w=r e^{i \theta}$ and by the subharmonicityof $\left|h^{\prime}\left(\varphi_{a}(w)\right) \varphi_{a}^{\alpha-1}(w) f^{\gamma}\left(\varphi_{a}(w)\right) \varphi^{\prime}{ }_{a}(w)\right|^{2}$, we obtain

$$
\begin{aligned}
\left\|I_{f}^{\gamma, \alpha}\right\|^{2} & \geq \sup _{a \in \mathbb{D}} \int_{0}^{1} \frac{1}{\pi} \int_{0}^{2 \pi}\left|h^{\prime}\left(\varphi_{a}\left(r e^{i \theta}\right)\right) \varphi_{a}^{\alpha-1}\left(r e^{i \theta}\right) f^{\gamma}\left(\varphi_{a}\left(r e^{i \theta}\right)\right) \varphi^{\prime}{ }_{a}\left(r e^{i \theta}\right)\right|^{2}\left(1-r^{2}\right)^{p} r d r d \theta \\
& \geq \sup _{a \in \mathbb{D}}\left|h^{\prime}(a) f^{\gamma}(a) a^{\alpha-1}\right|^{2}\left(1-|a|^{2}\right)^{2} \int_{0}^{1} 2\left(1-r^{2}\right)^{p} r d r \\
& =\frac{1}{1+p} \sup _{a \in \mathbb{D}}\left|h^{\prime}(a) f^{\gamma}(a) a^{\alpha-1}\right|^{2}\left(1-|a|^{2}\right)^{2} \\
& \geq \frac{1}{1+p}\left|h^{\prime}\left(z_{0}\right) f^{\gamma}\left(z_{0}\right) z_{0}^{\alpha-1}\right|^{2}\left(1-\left|z_{0}\right|^{2}\right)^{2} \\
& \geq \frac{1}{1+p} \frac{\left|f^{\gamma}\left(z_{0}\right) z_{0}^{\alpha-1}\right|^{2}}{\left\|g_{z_{0}}\right\|_{Q_{p}}^{2}} .
\end{aligned}
$$

By Lemma 2.1 we obtain

$$
\left\|I_{f}^{\gamma, \alpha}\right\| \geq\left|f^{\gamma}\left(z_{0}\right) z_{0}^{\alpha-1}\right|>C-\epsilon .
$$

Since $\epsilon$ is arbitrary, we have $\left\|I_{f}^{\gamma, \alpha}\right\| \geq \sup _{z \in \mathbb{D}}\left|f^{\gamma}(z) z^{\alpha-1}\right|$ and the proof is complete.

Theorem 2.2.Let $\gamma \leq 1$ and $\alpha \geq 1$. If $f \in Q_{p}$, then $J_{f}^{\gamma, \alpha}$ is bounded on $H^{\infty}$. Moreover $\left\|J_{f}^{\gamma, \alpha}\right\|_{Q_{p}} \leq\|f\|_{Q_{p}}$.

Proof. If $\|h\|_{H^{\infty}}=1$, then we have

$$
\begin{aligned}
&\left\|J_{f}^{\gamma, \alpha} h\right\|_{Q_{p}}^{2}=\sup _{a \in \mathbb{D}} \int_{\mathbb{D}}\left|h(z) z^{\alpha-1} f^{\prime \gamma}(z)\right|^{2}\left(1-\left|\varphi_{a}(z)\right|^{2}\right)^{p} d \sigma(z) \\
& \leq\|h\|_{H^{\infty}}^{2} \sup _{a \in \mathbb{D}} \int_{\mathbb{D}}\left|f^{\prime}(z)\right|^{2}\left(1-\left|\varphi_{a}(z)\right|^{2}\right)^{p} d \sigma(z) \\
& \leq\|h\|_{H^{\infty}}^{2}\|f\|_{Q_{p}}^{2}=\|f\|_{Q_{p}}^{2} .
\end{aligned}
$$

Therefore $J_{f}^{\gamma, \alpha}$ is bounded on $H^{\infty}$ and $\left\|J_{f}^{\gamma, \alpha}\right\|_{Q_{p}} \leq\|f\|_{Q_{p}}$. The proof is complete.

Theorem 2.3.Assume that $0<p<1, \gamma+\alpha \geq 1$ and $f \in \mathcal{A}$. Thenthe integral operator $I_{f}^{\gamma, \alpha}$ is compact from $Q_{p}$ space to $Q_{p}$ space if and only if $f \in H^{\infty}$.

Proof. If $I_{f}^{\gamma, \alpha}$ is compact, then it is bounded, and by Theorem 2.1 it follows that $f \in H^{\infty}$. Now assume $f \in H^{\infty}$ and $\left(h_{n}\right)$ is a sequence in $Q_{p}$ such that $h_{n} \rightarrow 0$. We have

$$
\begin{aligned}
\left\|I_{f}^{\gamma, \alpha} h_{n}\right\|_{Q_{p}}^{2} & =\sup _{a \in \mathbb{D}} \int_{D}\left|h_{n}^{\prime}(z) z^{\alpha-1} f^{\gamma}(z)\right|^{2}\left(1-\left|\varphi_{a}(z)\right|^{2}\right)^{p} d \sigma(z) \\
& \leq\left\|f^{\gamma}\right\|_{H^{\infty}}^{2}\left\|h_{n}\right\|_{Q_{p}}^{2} .
\end{aligned}
$$


Since for $h_{n} \rightarrow 0$ on $\overline{\mathbb{D}}$ we have $\left\|h_{n}\right\|_{Q_{p}} \rightarrow 0$, and by letting $\rightarrow \infty$ in the last inequality, we obtain thatlim ${ }_{n \rightarrow \infty}\left\|I_{f}^{\gamma, \alpha} h_{n}\right\|_{Q_{p}}=0$. Therefore, $I_{f}^{\gamma, \alpha}$ is compact.

A function $f \in \mathcal{H}(\mathbb{D})$ is called uniformly locally univalent if there exists a constant $\rho>0$ such that $f$ is univalent on the hyperbolic $\operatorname{disk}|(z-a) /(1-\bar{a} z)|<\tanh \rho$ of radius $\rho$ for every $a \in D$. It is known that a non-constant analytic function $f$ is uniformly locally univalent if and only if the norm

$$
\left\|f^{\prime \prime} / f^{\prime}\right\|=\sup _{z \in \mathbb{D}}\left(1-|z|^{2}\right)\left|\frac{f^{\prime \prime}(z)}{f^{\prime}(z)}\right|
$$

of the pre-Schwarzian derivative $f^{\prime \prime} / f^{\prime}$ of $f$ is finite. Let

$$
B(\lambda)=\left\{f \in \mathcal{H}(\mathbb{D}) ;\left\|f^{\prime \prime} / f^{\prime}\right\| \leq 2 \lambda\right\} .
$$

Kim and Sugawa in $[8,9]$ investigated various properties of the functions belong to the class $B(\lambda)$. The following Lemma is doue to Becker [3].

Lemma 2.2.If $f \in \mathcal{A}$ and $\left\|f^{\prime \prime} / f^{\prime}\right\| \leq 1$, then $f$ is univalent.

In the next theorem we prove:

Theorem 2.4. Let $0<\lambda<\alpha$ and $f \in B(\lambda)$. Then $f \in \mathbb{B}^{\alpha}$ and

$$
\|f\|_{\mathbb{B}^{\alpha}} \leq|f(0)|+\left|f^{\prime}(0)\right| 2^{\lambda+\alpha} .
$$

Proof. Let $0<\lambda<\alpha,|z|=r$ and $f \in B(\lambda)$. Then we have

$$
\begin{aligned}
\log \left|\frac{f^{\prime}(z)}{f^{\prime}(0)}\right| & \leq\left|\log \frac{f^{\prime}(z)}{f^{\prime}(0)}\right| \\
& =\left|\int_{0}^{z} \frac{f^{\prime \prime}(w)}{f^{\prime}(w)} d w\right| \\
& \leq r \int_{0}^{1}\left|\frac{f^{\prime \prime}(t z)}{f^{\prime}(t z)}\right| d t \\
& \leq r \int_{0}^{1} \frac{2 \lambda}{1-r^{2} t^{2}} d t \\
& =2 \lambda \log \sqrt{\frac{1+r}{1-r}}
\end{aligned}
$$

This implies

$$
\left|f^{\prime}(z)\right| \leq\left|f^{\prime}(0)\right|\left(\frac{1+r}{1-r}\right)^{\lambda}
$$


Therefore we have

$$
\begin{aligned}
& \|f\|_{\mathbb{B}^{\alpha}}=|f(0)|+\sup _{z \in \mathbb{D}}\left|f^{\prime}(z)\right|\left(1-|z|^{2}\right)^{\alpha} \\
& \leq|f(0)|+\left|\boldsymbol{f}^{\prime}(\mathbf{0})\right| \sup _{0<r<1}\left(1-r^{2}\right)^{\alpha}\left(\frac{\mathbf{1 + r}}{\mathbf{1}-\boldsymbol{r}}\right)^{\lambda} \\
& \leq|f(0)|+2^{\lambda+\alpha}\left|\boldsymbol{f}^{\prime}(\mathbf{0})\right| \sup _{0<r<1}(1-r)^{\alpha-\lambda} \\
& =|f(0)|+2^{\lambda+\alpha}\left|\boldsymbol{f}^{\prime}(\mathbf{0})\right|,
\end{aligned}
$$

and the proof is complete.

Theorem 2.5.Let $2 \lambda<1$ and $f \in \mathcal{A} \cap B(\lambda)$. Then $f \in Q_{p}$ for all $0<p<\infty$.

Proof.Let $2 \lambda<1$ and $f \in \mathcal{A} \cap B(\lambda)$. By setting $\alpha=1$ in Theorem 2.4, we have $f \in \mathbb{B}$. On the other hand, by Lemma 2.1, $f$ is univalent in the open unit disc and so $f \in S \cap \mathbb{B}$. Then by relation (1.2), $f \in S \cap Q_{p}$ for all $0<p<\infty . \square$

Corollary 2.1. Let $0<p<1, \gamma+\alpha \geq 1$ and $2 \lambda<1$. If $f \in H^{\infty}$, then for all $g \in B(\lambda), I_{f}^{\gamma, \alpha} g \in Q_{p}$ and $I_{f}^{\gamma, \alpha}$ is compact from $B(\lambda)$ space to $Q_{p}$ space.

\section{References}

[1] R. Aulaskari and P. Lappan, Criteria for an analytic function to be Bloch and a harmonic or meromorphic function to be normal,Complex Analysis and Its Applications, 305 (1994) 136-146.

[2] R. Aulaskari, P. Lappan, J. Xiao and R. Zhao, On $\alpha$-Bloch spaces and multipliers of Dirichlet spaces, J. Math.Anal.Appl. 209(1), (1997), 193-121.

[3] J. Becker, Lownersche Differentialgleichung und quasikonform fortsetzbare schlichte funktionen, J. reine Angew. Math. 255 (1972), 23-43.

[4] P. L. Duren, Theory of $H^{p}$ Spaces, Academic Press, New York, London(1970), Reprint:Dover, Mineola, New York (2000).

[5] B. J. Garnett, Bounded Analytic Functions, Graduate Texts in Mathematics, Springer, Berlin (2007). Revised first edition.

[6] D. Girela, Analytic functions of bounded mean oscillation, In: Aulaskari, R. (ed.) Complex Function Spaces, Mekrijarvi 1997. Univ. Joensuu Dept. Math. Rep. Ser., 4 (2001), 61-170.

[7] C. Hammond, The norm of a composition operator with linear symbol acting on the Dirichlet space, J. Math. Anal. Appl. 303 (2005), 499-508.

[8] Y. C. Kim and T. Sugawa, Growth and coefficient estimates for uniformly locally univalent functions on the unit disk, Rocky Mountain J. Math. 32 (2002), 179-200.

[9] Y. C. Kim and T. Sugawa, Uniformly locally univalent functions and Hardy spaces, J. Math. Anal. Appl. 353 (2009), 61-67.

[10] F. John and L. Nirenberg, On functions of bounded mean oscillation, Commun. Pure Appl. Math. 14 (1961), 415-426.

[11] H. Li and S. Li, Norm of an integral operators on some analytic function spaces on the unit disk, Journal of Inequalities and Applications, 342 (2013), 1-7.

[12] S. Li and S. Stevic, Integral type operators from mixed-norm spaces to $\alpha$-Bloch spaces. Integral Transforms Spec. Funct. 18(7), (2007) 485-493.

[13]Ch. Pommerenke, Schlichtefunktionen und analytische funktionen von Bechranktermittlereroszillation, Comment. Math. Helv. 52 (1977) 591-602. 
[14] D. Sarason, Function theory on the unit circle, Virginia Polytechnic Institute and State University. Blacksburg, Virginia (1978).

[15] S. Stevic, On an integral operator between Bloch-type spaces on the unit ball, Bull. Sci. Math. 134 (2010), 329-339.

[16] J. Xiao, Holomorphic Q Classes, Lecture Notes in Mathematics, vol. 1767. Springer, Berlin (2001).

[17] J. Xiao, Ceometric Q Functions, Frontiers in Mathematics, Birkhauser, Basel (2006).

[18] K. Zhu, Operator theory in function spaces, Marcel Dekker, New York (1990). Reprint: Math. Surveys and Monographs, vol. 138. American Mathematical Society, Providence (2007).

[19] K. Zhu, Bloch type spaces of analytic functions, Rocky Mt. J. Math. 23 (1993) 1143-1177. 